\section{Five new records of nematodes from East Antarctica}

\author{
P. Bohra ${ }^{1}$, A.K. Sanyal ${ }^{2}$, A. Hussain ${ }^{3}$ \& B. Mitra ${ }^{4}$
}

'Zoological Survey of India, Desert Regional Centre, Jhalamand, Pali Road, Jodhpur, Rajasthan 342005, India. ${ }^{284}$ Prani Vigyan Bhawan, Zoological Survey of India, M-Block, 535 New Alipore, Kolkata, West Bengal 700053, India. ${ }^{3}$ Zoological Survey of India, Northern Regional Centre, 218, Kaulagarh Road, Dehra Dun, Uttarakhand 248195, India. Email: ${ }^{1}$ bohrapadma@gmail.com

The terrestrial invertebrate fauna of Antarctica comprise of Protozoa, Rotifera, Tardigrada, Nematoda and Arthropoda. A handful of soil contains thousands of the microscopic nematode worms, many of them parasites of insects, plants or animals. Free-living species are abundant, including nematodes that feed on bacteria, fungi, and other nematodes.

The Antarctic region has been subdivided into three main ecological zones viz., the Continental Antarctic, the Maritime Antarctic and the Sub-Antarctic with distinctive climatic and biotic characteristics (Holdgate 1970). The free-living soil, freshwater and moss inhabiting nematodes of these regions have been studied by de Man (1904), Krijanova (1958), Timm (1971), Gray (1975), Loof (1975), Heyns (1993), Kito et al. (1996), and others. Andrassy (1998) has reported nematodes as the most abundant group in the Antarctic region. He listed 43 species comprising 29 species from the maritime region and 14 from Antarctica proper. Besides, he has also discussed survival strategies and geographic distribution

Date of publication (online): 26 June 2010

Date of publication (print): 26 June 2010

ISSN 0974-7907 (online) | 0974-7893 (print)

Editor: Anwar L. Bilgrami

Manuscript details:

Ms \# 02342

Received 09 November 2009

Final received 11 May 2010

Finally accepted 15 May 2010

Citation: Bohra, P., A.K. Sanyal, A. Hussain \& B. Mitra (2010). Five new records of nematodes from East Antarctica. Journal of Threatened Taxa 2(6): 974-977.

Copyright: @ P. Bohra, A.K. Sanyal, A. Hussain \& B. Mitra 2010. Creative Commons Attribution 3.0 Unported License. JoTT allows unrestricted use of this article in any medium for non-profit purposes, reproduction and distribution by providing adequate credit to the authors and the source of publication.

Acknowledgement: The authors are highly thankful to Dr. Ramakrishna, Director, Zoological Survey of India, Kolkata for providing necessary facilities to carry out the research work.

\section{OPEN ACCESS | FREE DOWNLOAD}

of nematodes. He has also provided a key to the species of Plectidae and Qudsianematidae. Bostrom (2005) added three species of nematodes from Maudland, East Antarctica and Ghosh et al. (2005) reported one new species Antarctenchus motililius from East Antarctica. Andrassy (2008) described six new species of the genus Eudorylaimus from continental Antarctica.

The moss and soil samples were collected from different sites at the periphery of Priyadarshini Lake (PDL) located at Schirmacher Oasis, East Antarctica. The nematodes were killed and fixed in hot $4 \%$ formalin and mounted in anhydrous glycerin. The specimens are deposited in the National Zoological Collection of Desert Regional Centre, Zoological Survey of India, Jodhpur.

\section{Results}

The analysis of samples yielded five species belonging to three genera and three families of the Orders Tylenchida, Dorylaimida and Araeolaimida. Helicotylenchus dihystera, $H$. diagonicus, $H$. exallus, Eudorylaimus sabulophilus and Plectus telekii are being reported for the first time from East Antarctica.

\section{Systematic account}

Order: Tylenchida Thorne, 1949

Superfamily: Hoplolaimoidea Filipjev, 1934

(Paramonov, 1967)

Family: Hoplolaimidae, Filipjev, 1934 (Wieser, 1953)

Genus: Helicotylenchus Steiner, 1945

\section{Helicotylenchus diagonicus Perry in Perry, Darling \& Thorne, 1959}

(Fig. 1)

Material examined: 3 females, 14.i.1999, coll. A.K. Sanyal, Reg.No. IV/1923.

Host: Moss at the bank of PDL.

Measurements: Female (3): $L=0.49-0.90 \mathrm{~mm} ; \mathrm{a}=$ $24-26 ; b=5.5-6.0 ; c=50-55 ; V={ }^{10-11} 56-62^{9-10}$

\section{Description:}

Female: Body spiral shaped. Lip region truncated with 3-4 annules. Stylet 20-22 $\mu \mathrm{m}$ long, basal knobs flattened slightly indented anteriorly. Spermatheca offset without sperms. Phasmids at the level of anus. Tail more curved dorsally with slight ventral projection.

Abbreviations: $\mathrm{L}=$ body length $(\mathrm{mm} / \mu \mathrm{m})$; $\mathrm{a}=$ body length / maximum body width; $b$ = body length / oesophageal length; $C$ = body length / tail length; $\mathrm{c}^{\prime}$ = tail length / body width at anus; $\mathrm{V}$ = distance from head end to vulva $\times 100 /$ body length 

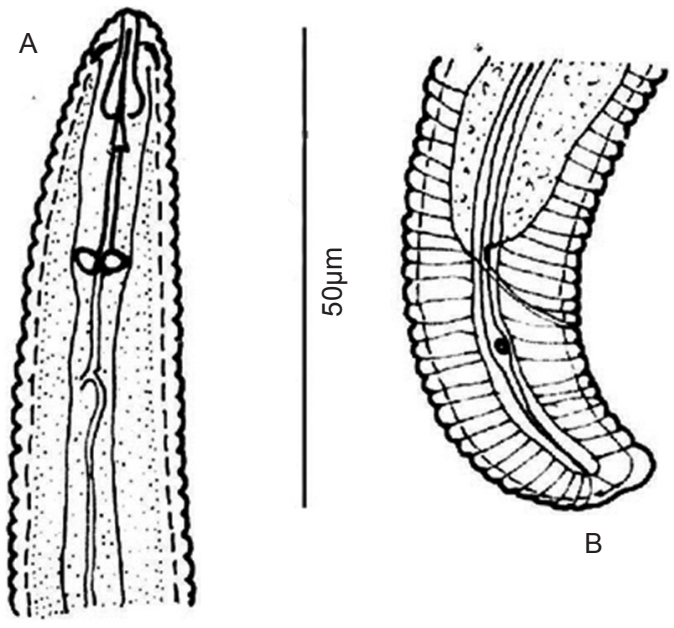

Figure 1. Helicotylenchus diagonicus A - Female Anterior region; B - Posterior region.

Male: Not found.

Distribution: Canada, Greece, India, Poland, USA.

Remarks: First report from Antarctica.

\section{Helicotylenchus exallus Sher, 1966}

(Fig. 2)

Material examined: 5 females, 30.xii.1999, coll. A.K. Sanyal, Reg.No. IV/1924.

Host: Moss at the bank of PDL.

Measurements: Females (5): $\mathrm{L}=0.61-0.65 \mathrm{~mm} ; \mathrm{a}=$ $26-28 ; b=6.0-6.2 ; c=40-45 ; c^{\prime}=0.7-1.2 ; V={ }^{10-11} 60-62^{8}$ 10

\section{Description}

Female: Body spiral shaped. Lip region hemispherical marked by four annules. Stylet $24-26 \mu \mathrm{m}$ long, basal knobs with flattened anterior surface. Spermatheca large, offset with sperms. Phasmid 2-3 annules anterior to anus. Tail more curved dorsally with slight ventral projection.

Male: Not found.

A
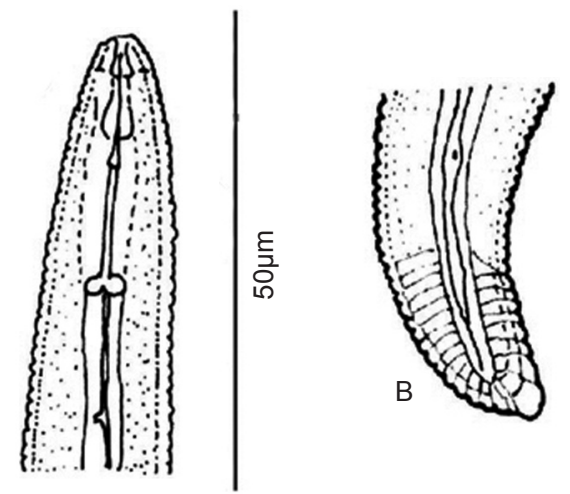

Figure 2. Helicotylenchus exallus A - Female Anterior region; B - Posterior region.
Distribution: India, USA.

Remarks: First report from Antarctica.

Helicotylenchus dihystera (Cobb, 1893) Sher, 1961 (Fig. 3)

Material examined: 5 females, 03.i.1999, coll. B. Mitra, Reg.No. IV/1921.

Host: Wet Moss along with soil at the bank of Priyadarshini Lake (PDL).

Measurements: Females (5): $L=0.54-0.83 \mathrm{~mm} ; \mathrm{a}=$ $20-32 ; b=4.5-6.4 ; c=44-46 ; c^{\prime}=1.0-1.3 ; \quad V={ }^{12-15} 60$ $66^{11-13}$

\section{Description}

Female: Body spiral. Cuticle with distinct transverse striae. Lip region continuous with body, hemispherical bearing four annules. Cephalic framework conspicuous. Lateral fields marked by four incisures. Stylet 24-26 $\mu \mathrm{m}$ long, basal knobs concave. Oesophagus with gland overlapping ventrally. Spermatheca rounded offset, without sperms. Tail dorsally convex-conoid usually with slight ventral projection.

Male: Not found.

Distribution: This is a cosmopolitan and widely distributed species.

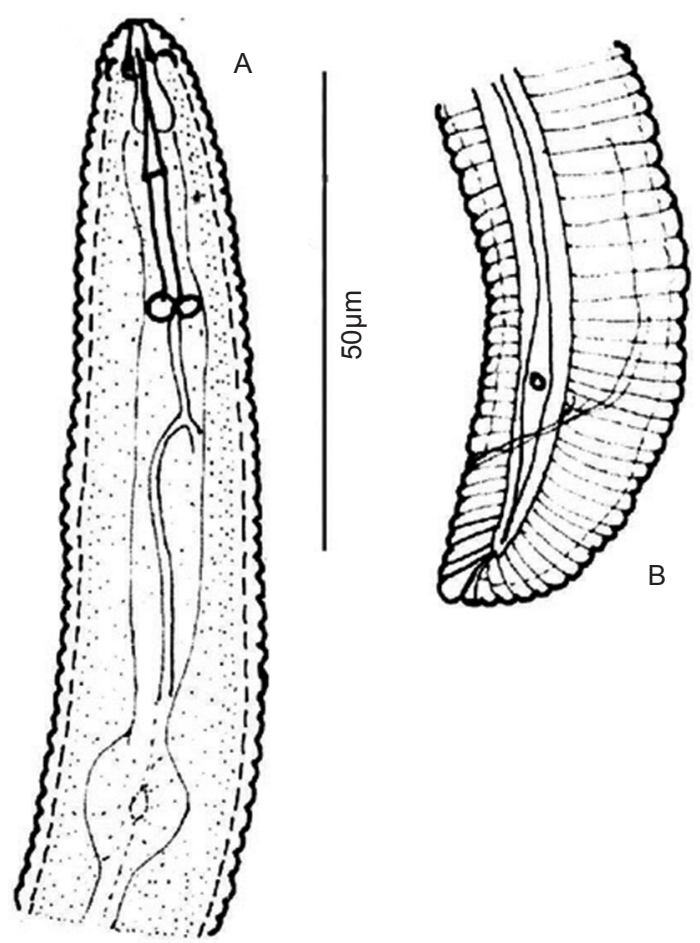

Figure 3. Helicotylenchus dihystera

A - Female Anterior region; B - Posterior region. 

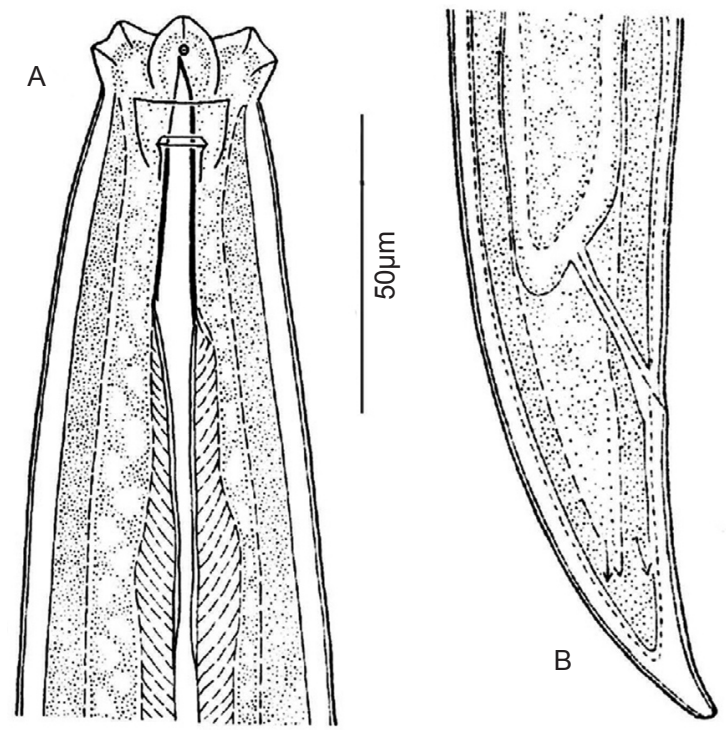

Figure 4. Eudorylaimus sabulophilus

A - Female Anterior region; B - Female Posterior region.

Remarks: First time reported from Antarctica.

Order: Dorylaimida Pearse, 1942

Superfamily: Dorylaimoidea De Man, 1876

Family: Qudsianematidae Jairajpuri, 1965

\section{Eudorylaimus sabulophilus Tijepkema, Ferris \& Ferris, 1971}

(Fig. 4)

Material examined: 3 females, 23.ii.1999, coll. A. Hussain, Reg.No. IV/2240

Host: Moss and algae at the bank of PDL.

Measurement: Females (3): $L=0.87-0.98 \mathrm{~mm} ; a=$ $17-22 ; b=3.47-3.92 ; c=17.3-21.7 ; \mathrm{V}=56-59$.

\section{Description:}

Female: Body slightly curved ventrally upon fixation. Lips well separated and set off from body by constriction. Odontostyle 17-18 $\mu \mathrm{m}$ long; its aperture $30 \%$ of odontostyle length. Odontophore rod-like 22-24 $\mu \mathrm{m}$ long. Oesophageal expansion gradual. Basal expanded part of oesophagus occupies $45-47 \%$ of oesophageal length. Reproductive system amphidelphic. Tail dorsally-convex with subacute or narrow rounded tip. Tail tip slightly bent ventrally.

Male: Not found.

Distribution: USA.

Remarks: First time reported from Antarctica.
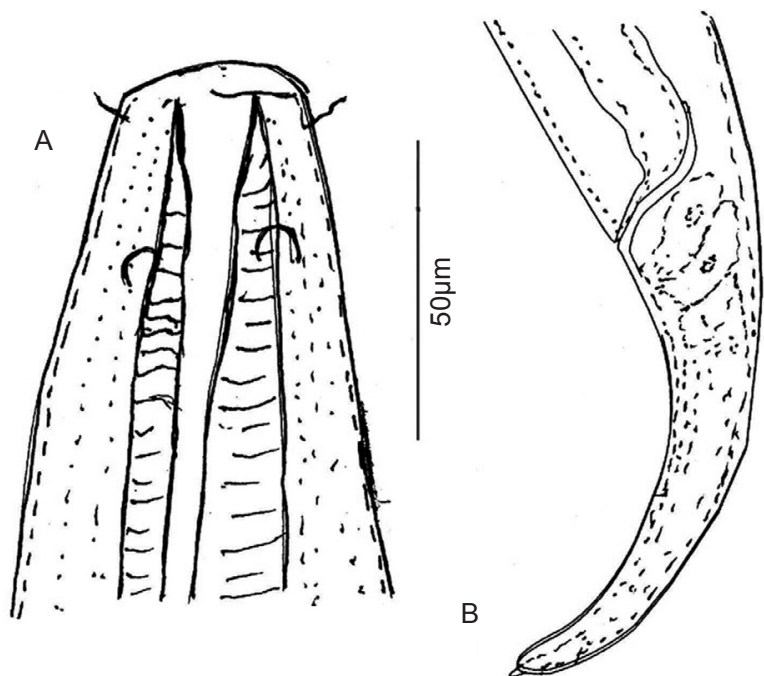

Figure 5. Plectus telekii

A - Female Anterior region; B - Female Posterior region.

\section{Order: Araeolaimida De Coninck \& Schuurmans} Stekhoven, 1933

Superfamily: Plectoidea Örley, 1880

Family: Plectidae Örley, 1880

\section{Plectus telekii Mulk \& Coomans, 1978}

(Fig. 5)

Material examined: 6 females, 23.ii.1999, coll. A. Hussain, Reg.No. IV/1925-26.

Host: Moss at the bank of PDL.

Female (5): $\mathrm{L}=669 \mu \mathrm{m}-794 \mu \mathrm{m} \mathrm{a}=18.0-19.0, \mathrm{~b}=$ 4.0-4.5, c=8.0-8.8, c' = 3.7-5.0, V = 45.2-49.7, Pharynx = $165-186 \mu \mathrm{m}$, Anal body diameter $=18-22 \mu \mathrm{m}$, Tail length $=79-97 \mu \mathrm{m}$

\section{Description:}

Female: Body medium-sized, arcuate, open C shape upon fixation. Lip region continuous with body contour. Lips elevated 2.5-3.0 $\mu \mathrm{m}$ long. Lip region 7-11 $\mu \mathrm{m}$ wide. Cephalic sensilla setose, 2.0-2.5 $\mu \mathrm{m}$ long. Amphids located 11-14 $\mu \mathrm{m}$ from anterior end. Stoma 19-30 $\mu \mathrm{m}$ long, cheilostom cuticularised. Nerve ring encircles isthmus. Excretory pore inconspicuous. Intestine granular. Female reproductive system amphidelphic. Ovary reflexed. Vulva slit like. Uterus without egg. Vulva-anus distance three times tail length. Tail cylindrical, arcuate. Spinneret about $2 \mu \mathrm{m}$ long.

Male: Not found.

Distribution: Rajasthan, India

Remarks: First report from Antarctica. 


\section{REFERENCES}

Andrassy, I. (1998). Nematodes in Sixth continent. Journal of Nematode Morphology and Systematics 1: 107-186.

Andrassy, I. (2008). Eudorylaimus species (Nematoda: Dorylaimida) of continental Antarctica. Journal of Nematode Morphology \& Systematic 11(1): 49-66.

Bostrom, S. (2005). Nematodes from Sirmacher Oasis, Dronning, Maud Land, East Antarctica. Russian Journal of Nematology 13: 43-54.

Christy, J.R. \& V.G. Perry (1951). Removing nematodes from soil. Proceedings Helminthological Society, Washington 18 106-108.

Cobb, N.A. (1918). Estimating the Nema Population of the Soil. Agricultural Technology Circular I. Bureau of Plant Industry, United States, Department of Agriculture, 48pp.

De Man, J.G. (1904). Nematodes libres. Result. Voyage S.Y. Belegica, Zoologie 55pp.

Ghosh, S.C, A.C. Bulgarin \& J. De (2005). Antarctenchus motililus sp. n. (Nematoda: Tylenchida) from Schirmacher Oasis, East Antarctica. Journal of Interacademicia 9(3): 367371.

Gray, N.F.(1975).Apreliminary survey ofAntarcticnematophagous fungi with reference to temperature tolerance. BSc. Degree Project Report No. 745/1138. The Polytechnic of Central London, 92pp (unpublished).
Heyns, J. (1993). Eudorylaimus nudicaudatus sp. n. from Antarctica (Nematoda: Dorylaimoidea). South African Journal of Antarctic Research 23: 33-36.

Holdgate, M.W. (ed.) (1970). Antarctic Ecology. London and New York; Academic Press, 998pp (2 volumes). [Proceedings of $2^{\text {nd }}$ SCAR Symposium on Antarctic Biology]

Kito, K., Y. Shishida \& Y. Ohyama (1996). A new species of the genus Eudorylaimus Andrassy, 1959 (Nematoda: Qudsianematidae) from East Antarctica. Polar Biology 16: 163-169.

Krijanova,E.S.(1958).Antarkticheskie predsatavitelipredstaviteli presnovodnykh nematodroela Plectus Bastian (Nematodes, Plectidae) (Antarctic specimens of freshwater nematodes of the genus Plectus Bastian (Nematoda, plectida). Inf. Byull. Sov. Antarkt. Eksped. No. 3 : 101-03 [English translation Vol. $1,1964]$

Loof, P.A.A. (1975). Dorylaimoidea from some subantarctic islands. Nematologica 21: 219-255.

Seinhorst, J.W. (1959). A rapid method for the transfer of nematodesfrom fixative to anhydrous glycerine. Nematologica 4: 67-69.

Timm, R.W. (1971). Antarctic soil and freshwater nematodes from the Mc Murdo Sound Region. Proceedings of the Helminthological Society of Washington 38: 42-52. 